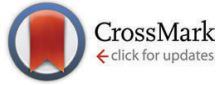

Cite this: Phys. Chem. Chem. Phys., $2015,17,24356$

Received 2nd June 2015, Accepted 17th August 2015

DOI: $10.1039 / c 5 c p 03189 c$

www.rsc.org/pccp

\section{Molecularly linked 3D plasmonic nanoparticle core/satellite assemblies: SERS nanotags with single-particle Raman sensitivity $\dagger$}

\author{
Max Schütz and Sebastian Schlücker*
}

\begin{abstract}
A fast, generic, and suspension-based route to highly SERS-active assemblies of noble metal nanoparticles (Au, Ag) with small core-satellite gaps and single-particle Raman sensitivity is presented. Rationally designed, heterobifunctional Raman reporters serve as molecular linkers for electrostatic conjugation of the small satellites to the large core.
\end{abstract}

\section{Introduction}

Noble metal colloids support localized surface plasmon resonances (LSPR) upon resonant excitation with light. ${ }^{1-3}$ Very high local electromagnetic field enhancements ("hot spots") are observed at sharp tips ${ }^{4}$ and at junctions between two nanoparticles (NPs)..$^{5-8}$ Surface-enhanced Raman scattering (SERS) ${ }^{9,10}$ from molecules located in the hot spot can be employed for optically probing the corresponding high local field enhancements. ${ }^{11,12}$

The rational design of plasmonic NP assemblies with defined interparticle junctions and high, reproducible SERS signals has recently gathered large interest. ${ }^{13-15}$ The assembly process can be mediated and efficiently controlled by molecular linkers. ${ }^{16}$ Examples are biomacromolecules with recognition elements such as DNA ${ }^{17,18}$ as well as molecules comprising two terminal surface-seeking groups such as dithiols ${ }^{19}$ or diamines. ${ }^{20}$

Nanodiagnostic applications of highly SERS-active NPs for the selective detection and localization of analytes exploit the characteristic vibrational Raman signature of reporter molecules on the metal surface. ${ }^{21-23}$ The nanofabrication of highly SERS-active NP assemblies requires molecular linkers with large Raman scattering cross sections in the core-satellite gap. Ideally, the resulting molecularly linked $3 \mathrm{D}$ assemblies exhibit single-particle (single-assembly) SERS sensitivity as demonstrated by correlative SEM/SERS experiments.

Our initial approach towards 3D assemblies of AuNPs with single-particle SERS sensitivity was based on electrostatic binding of negatively charged, citrate-stabilized satellite NPs onto a

University of Duisburg-Essen, Faculty of Chemistry, Center for Nanointegration Duisburg-Essen (CENIDE), Universitätsstr. 5, 45141 Essen, Germany.

E-mail: sebastian.schluecker@uni-due.de; Fax: +49 2011836841; Tel: +492011836843

$\dagger$ Electronic supplementary information (ESI) available: Further data. See DOI: 10.1039/c5cp03189c positively charged core NP coated with an amino-functionalized thin silica shell. ${ }^{13} \mathrm{~A}$ central disadvantage of this approach is the relatively large core-satellite gap $(>2 \mathrm{~nm})$ due to the silica shell, which reduces the plasmonic enhancement, and also the requirement of several surface modification steps. Here, we present a simple yet generic route to core/satellite assemblies with a small gap (estimated 1-2 $\mathrm{nm}$ ) using rationally designed heterobifunctional Raman reporters as molecular linkers.

\section{Results and discussion}

\subsection{Synthesis}

Specifically, we employ 5,5'-dithiobis(2-nitrobenzoic acid) (DTNB) as the actual Raman reporter and conjugate it to a bisamino-diethylene glycol $\left(\mathrm{H}_{2} \mathrm{~N}-\mathrm{DEG}-\mathrm{NH}_{2}\right)$ for both hydrophilic stabilization (ethylene glycol) 24,25 $^{24}$ and subsequent electrostatic conjugation (amino group). ${ }^{26,27}$ Upon incubation with $80 \mathrm{~nm}$, PVP-capped AuNP cores, the conjugate (DTNB-DEG-NH $\mathrm{N}_{2}$ ) forms a Raman-active (DTNB), positively charged (terminal $-\mathrm{NH}_{3}{ }^{+}$) and hydrophilically stabilized (DEG) self-assembled monolayer (SAM), which replaces PVP by the formation of Au-S bonds ${ }^{28}$ (Fig. 1 top). Electrostatic binding of negatively charged, citratestabilized AuNP satellites (zeta potential up to $-30 \mathrm{mV}$ ) onto the positively charged core (zeta potential up to $+20 \mathrm{mV}$ ) leads to $3 \mathrm{D}$ core/satellite assemblies, ${ }^{27}$ which are subsequently encapsulated by a silica shell (Fig. 1 bottom) for chemical and mechanical stabilization, using carboxyethyl silanetriol and tetraethylorthosilicate (TEOS) in a modified Stöber synthesis. ${ }^{29,30}$

Monomeric noble metal nanoparticles were synthesized according to existing protocols. Briefly, small AuNPs for use as satellites were synthesized by reduction of tetrachloroauric(III) acid with sodium citrate. These particles were also used as seeds to grow bigger AuNPs with $\sim 80 \mathrm{~nm}$ in diameter by reduction 


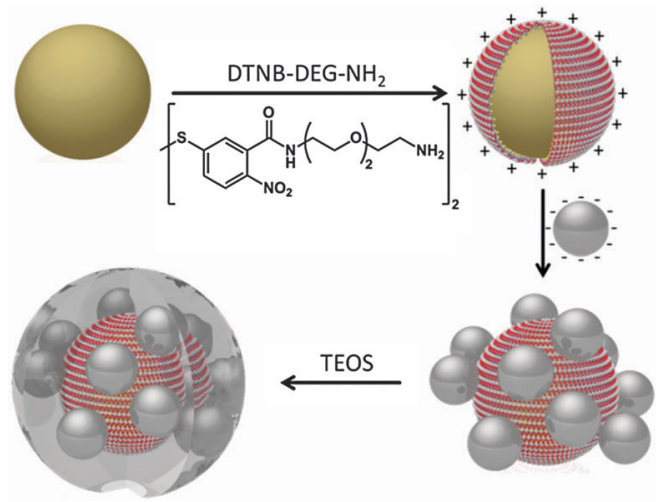

Fig. 1 Synthesis route to silica-encapsulated Ag@Au 3D superstructures, starting from PVP-coated gold nanospheres. After formation of the selfassembled monolayer, citrate-stabilized silver nanospheres are added and afterwards encapsulated using TEOS.

with hydroquinone for use as core particles. ${ }^{31}$ Monodisperse $\mathrm{Ag}$ cores and satellites were synthesized by reduction of silver nitrate in water/glycerol. ${ }^{32}$ More details on all syntheses can be found in the section on Materials and methods.

In order to saturate the entire surface of each core with satellites, it is important to employ a very low concentration of core particles in the presence of a large excess of satellites. Due to electrostatic repulsion between the resulting 3D core/satellite superstructures, uncontrolled aggregation does not occur.

The core-satellite gap distance is estimated to be $1-2 \mathrm{~nm}$, based on the length of the heterobifunctional Raman reporter and linker molecule DTNB-DEG-NH $\mathrm{N}_{2}$ on the core as well as the thickness of the citrate shell on the satellites. The length of DTNB-DEG- $\mathrm{NH}_{2}$ is a conservative estimation and therefore an upper limit since it is based on an all-trans conformation of the conformationally flexible DEG- $\mathrm{NH}_{2}$ subunits attached to the planar phenyl rings. A precise experimental demonstration of the gap size requires high-resolution TEM, which was not accessible to us when the characterization of the particles was performed. Overall, this approach requires no additional (thicker) silica shell for the encapsulation of the core as in our earlier work $^{13}$

It is very important to use PVP-capped core particles and citrate-stabilized satellites. Using citrate stabilized cores results in an immediate uncontrolled aggregation upon addition of DTNB-DEG- $\mathrm{NH}_{2}$. On the other hand, PVP-capped satellites are not as good for forming assemblies as citrate-capped satellites are. These observations can be explained by the surface affinity of the bifunctional crosslinker. In the case of PVP-capped core particles, the crosslinker binds via the thiol group to the gold surface of the core particles. The terminal amino groups of the resulting positively charged cores $\left(\mathrm{NH}_{3}{ }^{+}\right)$have a high affinity to the negatively charged, citrate-stabilized satellite particles. In contrast, the affinity to PVP-capped satellites is significantly lower. The uncontrolled aggregation observed upon the addition of DTNB-DEG-NH $\mathrm{N}_{2}$ to citrate-capped core particles is attributed to charge neutralization and/or binding between two cores at once.

The presented approach is not limited to AuNP cores and AuNP satellites. We synthesized various $3 \mathrm{D}$ noble metal NP assemblies using either AgNPs or AuNPs as core particles in combination with smaller AgNPs or AuNPs as satellites. The ethylene glycol units provide stability in aqueous suspension and generate a robust SAM-coated core particle. ${ }^{24,25}$ Important is only the capping agent: PVP for the core particles and citrate for the satellites. Four combinations are possible: gold core and silver satellites (Ag@Au), gold core and satellites (Au@Au), silver core and satellites (Ag@Ag) as well as gold core and silver satellites (Ag@Au).

\subsection{Characterization}

We obtained all four types of assemblies and characterized them by TEM. Fig. 2 displays TEM images of different noble metal NP assemblies prior to (A-D) and after silica coating ( $\mathrm{E}$ and $\mathrm{F}$ ). The obtained particles are relatively homogeneous and the number of satellites on each core particle is more or less similar. Fig. 3 contains more TEM images (bottom) and also the UV/Vis extinction spectrum (top) for the Ag@Ag superstructures. TEM images and extinction spectra of the other three types of superstructures can be found in the ESI $\dagger$ (Fig. S1-S4).

The number of satellites assembled onto each core cannot be quantitatively determined since the TEM images do not give a complete 3D picture of the surface coverage. The plasmon peaks for all four superstructures exhibit maxima between $588 \mathrm{~nm}$ (Ag@Ag) and $632 \mathrm{~nm}(\mathrm{Au} @ \mathrm{Au})$, revealing the plasmonic coupling between the core and the satellites. In order to confirm the plasmonic coupling between core and satellites via their anticipated high SERS activity, the 3D plasmonic assemblies were investigated in correlative single-particle SEM/SERS experiments. The results are displayed in Fig. 4. First, SEM images were recorded (Fig. 4A) from assemblies in an area where no other
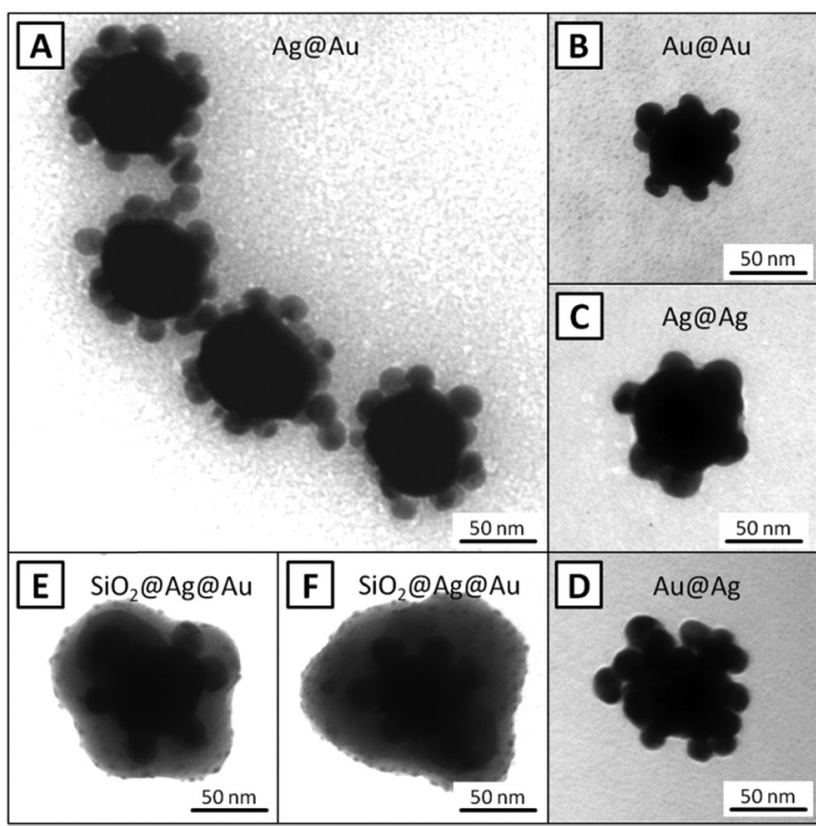

Fig. 2 TEM images of different 3D superstructures: Ag@Au (A), Au@Au (B), Ag@Ag (C), Au@Ag (D) and also silica-encapsulated Ag@Au (E and F). See $\mathrm{ESI} \uparrow$ for more images. 

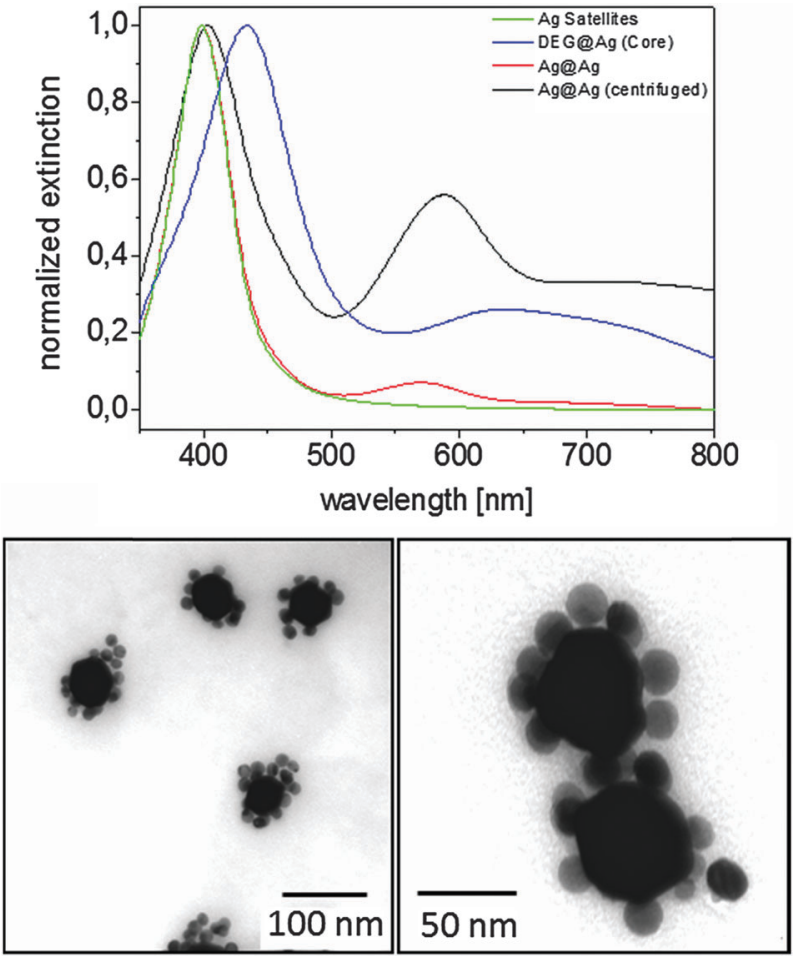

Fig. 3 (top) Normalized UV-VIS extinction spectra of Ag@Ag 3D superstructures (red: directly after the synthesis; black: after centrifugation and redispersion in ethanol) and their precursors (green: Ag satellites; blue: DTNB-DEG-covered core particles). The plasmon band of the 3D superstructures in ethanol is $\lambda_{\max }=588 \mathrm{~nm}$. (bottom) Display TEM images of the Ag@Ag 3D superstructures. See ESI $\dagger$ for more images.

NPs were present. In the next step the same area was investigated by confocal Raman microspectroscopy with 1s integration time and $1 \mathrm{~mW}$ laser power. The set of Raman spectra was used to generate a false color image (Fig. 4C) based on the intensity of the dominant Raman band of DTNB at $\sim 1340 \mathrm{~cm}^{-1}$, as highlighted in the SERS spectrum of the 3D assembly (marked with a cross in Fig. 4C) in Fig. 4B.

Our results show an intense Raman signal at the single-particle level, which is significantly stronger than in our previous work ${ }^{13}$ and therefore supports the conclusion on the presence of a shorter gap. A direct quantitative comparison, however, is not possible due to differences in the experimental conditions, in particular acquisition times, size of core and satellites as well as the number of satellites.

In summary, a simple, fast, generic and suspension-based synthesis of rationally designed silica-encapsulated and spectrally encoded 3D plasmonic assemblies is presented. The approach is based on a heterobifunctional crosslinker which also serves as the Raman reporter and is present as a SAM on the surface of the core $\mathrm{NP}(\mathrm{Au}$ or Ag). Upon addition of smaller satellite particles (Au or $\mathrm{Ag}$ ), the assemblies form immediately due to molecularly assisted self-assembly using Coulomb attraction. In contrast to solid phase-based approaches, ${ }^{27}$ this suspension-based approach leads to $3 \mathrm{D}$ plasmonic superstructures comprising cores located not only on a single hemisphere. In contrast to NP dimers, assemblies of NPs exhibit a quasi-polarization-independent SERS
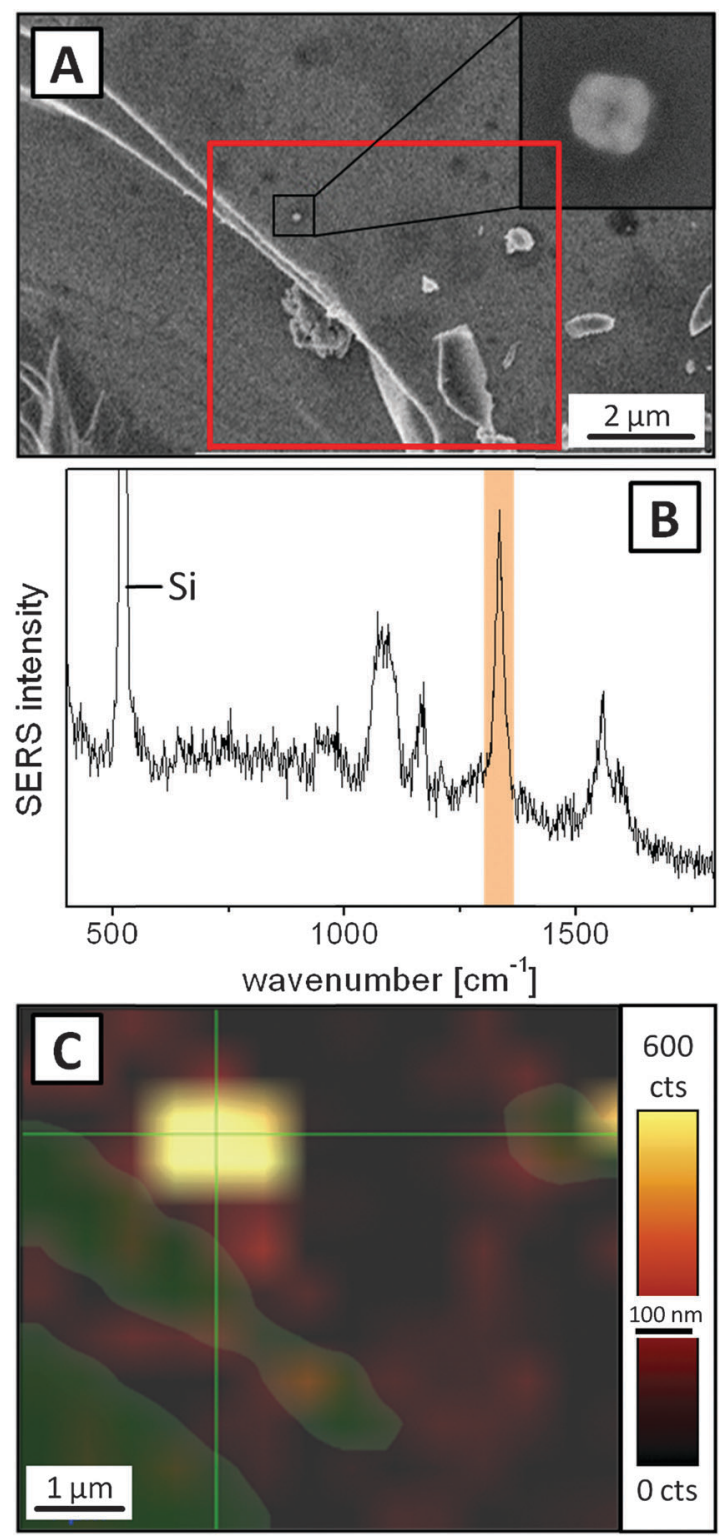

Fig. 4 Combined SEM/SERS measurement: (A) SEM image of an Ag@Au 3D superstructure including an inset with higher magnification (red rectangle shows the rough mapping area), (B) SERS spectrum of the single superstructure and (C) SERS false color image using the sum between 1290 and $1380 \mathrm{~cm}^{-1}$ as indicated in (B) and overlayed intensity of Si (green) showing the edge observed in (A).

response at the single-particle level. ${ }^{33}$ In further studies the silicaencapsulated 3D superstructures may be used as SERS labels in bioanalytical and biomedical applications, e.g. in SERS microscopy for tissue-based cancer diagnostics.

\section{Materials and methods}

\section{Syntheses}

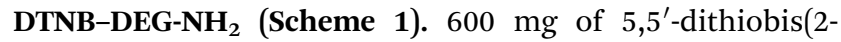
nitrobenzoic acid) (1.5 mmol) were dissolved in $10 \mathrm{ml}$ dry DCM. $1800 \mathrm{mg}$ Pybop (3.5 mmol), $700 \mathrm{mg}$ 4-(dimethylamino)pyridine 
( $5.7 \mathrm{mmol}$ ) and two drops of dry DMF were added. $735 \mu \mathrm{l}$ tert-butyl 2-[2-(2-aminoethoxy)ethoxy]ethylcarbamate (769 $\mathrm{mg}, 3.1 \mathrm{mmol}$ ) were slowly added. After stirring for 4 days, the solution was extracted twice with $50 \mathrm{ml}$ of $0.1 \mathrm{M} \mathrm{HCl}$ to remove DMAP and the organic solvent was dried with $\mathrm{MgSO}_{4}$. The product was isolated using a MPLC (eluent: DCM and methanol) and afterwards the Boc group was cleaved in $5 \mathrm{ml}$ DCM and $1 \mathrm{ml}$ TFA while stirring over night. The crude product was extracted with $1 \mathrm{M} \mathrm{HCl}$ and afterwards purified with C18-reversed phase (eluent: $\mathrm{H}_{2} \mathrm{O}$ and $\mathrm{MeOH}$ ) and normal phase MPLC (eluent: $\mathrm{MeOH})$.

${ }^{1} \mathrm{H}-\mathrm{NMR}\left(500 \mathrm{MHz}, \mathrm{CDCl}_{3}\right) \delta[\mathrm{ppm}]=3.16\left(\mathrm{~m}, 2 \mathrm{H}, \mathrm{CH}_{2}\right) ; 3.58$ (m, 4H, $\left.\mathrm{CH}_{2}\right) ; 3.69\left(\mathrm{~m}, 6 \mathrm{H}, \mathrm{CH}_{2}\right) 7.71$ (s, 1H, arom.); $7.86(\mathrm{~d}, 1 \mathrm{H}$, arom.); 8.04 (d, $1 \mathrm{H}$, arom.); $8.74\left(\mathrm{~s}, 1 \mathrm{H}, \mathrm{NH}-\mathrm{CH}_{2}\right) . \mathrm{m} / z\left(\mathrm{H}^{+}\right)$: $657.20 \mathrm{u}$ (calc: $657.2012 \mathrm{u}$ ).

Au nanoparticles $15 \mathrm{~nm}$ (satellites/seeds), $\lambda_{\max }=518 \mathrm{~nm}$. Small Au nanoparticles for use as satellites or as seeds to yield bigger nanoparticles were prepared according to literature protocols. ${ }^{31}$ Briefly, $300 \mu \mathrm{l}$ of $1 \%(\mathrm{w} / \mathrm{v}) \mathrm{HAuCl}_{4}$ and $900 \mu \mathrm{l}$ of $1 \%(\mathrm{w} / \mathrm{v})$ sodium citrate were added to $30 \mathrm{ml}$ of boiling water under vigorous stirring. After 5 minutes the solution was allowed to cool down and was slowly stirred overnight.

Au nanoparticles $80 \mathrm{~nm}$ (cores), $\lambda_{\max }=540 \mathrm{~nm}$. Starting from the seed solution a modified synthesis ${ }^{31}$ was used to yield PVP-capped Au nanoparticles. 250 mg PVP K30 were dissolved in $15 \mathrm{ml}$ ultrapure water and $500 \mu \mathrm{l}$ tetrachloroauric(III) acid solution (1\%), $1 \mathrm{ml}$ seed solution as well as $10 \mathrm{ml}$ hydroquinone solution (1.65 mg hydroquinone) were added at $20{ }^{\circ} \mathrm{C}$ while rapidly stirring to yield PVP-capped nanoparticles with $80 \mathrm{~nm}$ in diameter.

Ag nanoparticles $20 \mathrm{~nm}$ (satellites), $\lambda_{\max }=398 \mathrm{~nm}$. Small Ag nanoparticles for use as satellites were prepared as previously published. $^{32} 50 \mathrm{ml}$ water/glycerol (50 vol\% glycerol) were heated to $95{ }^{\circ} \mathrm{C}$ and stirred at $1200 \mathrm{rpm} .6 \mathrm{mg}$ silver nitrate and one minute later $1 \mathrm{ml}$ of sodium citrate solution $(3 \% \mathrm{w} / \mathrm{v})$ were added. After stirring for one hour at $95{ }^{\circ} \mathrm{C}$ the suspension was allowed to cool down.

Ag nanoparticles $80 \mathrm{~nm}$ (cores), $\lambda_{\max }=430 \mathrm{~nm}$. Silver nanoparticles with $80 \mathrm{~nm}$ in diameter were prepared as reported. ${ }^{32}$ First, seeds with $30 \mathrm{~nm}$ in diameter were synthesized by adding $9 \mathrm{mg}$ silver nitrate into $50 \mathrm{ml}$ water/glycerol $(40 \%$ glycerol) at $95{ }^{\circ} \mathrm{C}$. One minute later $1 \mathrm{ml}$ of sodium citrate solution $(3 \% \mathrm{w} / \mathrm{v})$ was added and stirred for one hour at $95{ }^{\circ} \mathrm{C}$. The seeds were used in a growth process to obtain monodisperse silver nanoparticles with $80 \mathrm{~nm}$ in diameter. $138 \mathrm{ml}$ water, $23 \mathrm{ml}$

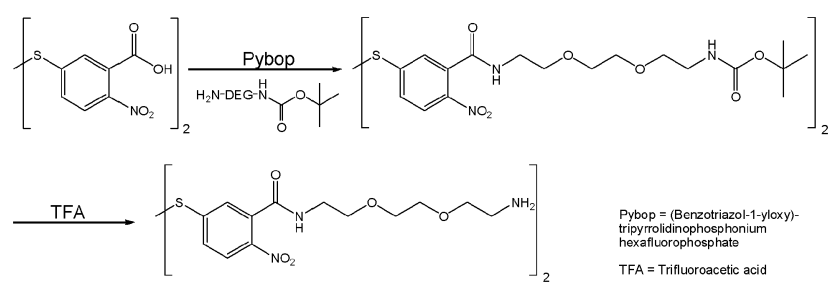

Scheme 1 Synthesis of DTNB-DEG-NH 2 starting from 5,5'-dithiobis(2nitrobenzoic acid). After activation of the carboxylic acid with Pybop, the diamine (one amino group is protected with boc) is added and the desired product can be obtained after deprotection using TFA. glycerol and $0.58 \mathrm{~g}$ PVP K30 were stirred at RT and $4.5 \mathrm{ml}$ seed solution was added. 20 seconds later $1.15 \mathrm{ml}$ diamine silver complex solution ( $20 \mathrm{mg}$ silver nitrate in $1 \mathrm{ml}$ water plus $220 \mu \mathrm{l}$ ammonium hydroxide 30\%) was added together with $92 \mathrm{ml}$ ascorbic acid solution (36.8 $\mathrm{mg}$ ascorbic acid). Finally, $6 \mathrm{~g}$ PVP were added after one hour.

3D superstructures. The $\mathrm{Ag}$ nanoparticles $(80 \mathrm{~nm})$ were centrifuged (15 min, $1920 \mathrm{~g}$ ) and incubated for $30 \mathrm{~min}$ in a PVP-water solution $(1 \mathrm{~g} / 40 \mathrm{ml})$. The Au nanoparticles were used as obtained during the synthesis. $4.5 \mathrm{ml}$ PVP-capped nanoparticles (80 nm Au or Ag) were centrifuged (15 min, $1920 \mathrm{~g}$ ) and dispersed in $700 \mu \mathrm{l}$ ultrapure water and $700 \mu \mathrm{l}$ 2-propanol. $50 \mu 10 \mathrm{mM}$ DTNB-DEG-NH $\mathrm{NH}_{2}$ solution (in 2-propanol) was added to this suspension. The samples were incubated for $4 \mathrm{~h}$ (or overnight), centrifuged afterwards, washed and dispersed in $500 \mu \mathrm{l}$ water. Meanwhile $12 \mathrm{ml}$ satellite particles (Ag or Au) were centrifuged (15 min, $7700 \mathrm{~g}$ ) and concentrated in $500 \mu \mathrm{l}$ water with $0.1 \%$ sodium citrate.

The 3D superstructures form immediately by adding for instance $100 \mu$ l of the concentrated seed particle suspension to $50 \mu \mathrm{l}$ of the SAM-functionalized core particles in water. Afterwards the suspension was slowly centrifuged (15 min, $250 \mathrm{~g}$ ) to avoid unwanted aggregation and the obtained superstructures were redispersed in ethanol for instance. The samples can be washed more often in order to remove all satellite particles. However, this is not mandatory for encapsulation.

Silica-encapsulation of the $3 \mathrm{D}$ superstructures. The $3 \mathrm{D}$ superstructures can be encapsulated using a modified Stöber synthesis. ${ }^{29}$ After centrifugation the Ag@Au 3D superstructures were suspended in $1000 \mu \mathrm{l}$ 2-propanol and $360 \mu \mathrm{l}$ ultrapure water. Then $10 \mu \mathrm{l}$ of carboxyethylsilanetriol solution (1\% in water) was added. After the addition of $20 \mu \mathrm{l}$ ammonium hydroxide solution (30\%) 10 minutes later, TEOS $(1 \%$ in 2-propanol) was added in small portions of $10 \mu \mathrm{l}$ in intervals of $1 \mathrm{~h}$. The shell thickness can be tuned by the amount of TEOS. Afterwards the encapsulated nanoparticles can be washed and stored after suspending them in ethanol.

\section{Materials}

Sigma-Aldrich: 5,5'-dithiobis(2-nitrobenzoic acid) (DTNB), dichloromethane (DCM), (benzotriazol-1-yloxy)tripyrrolidi-nophosphonium hexafluorophosphate (Pybop), 4-(dimethyl-amino)pyridine (DMAP), tert-butyl 2-[2-(2-aminoethoxy) ethoxy]ethylcarbamate (DEG- $\left.\mathrm{NH}_{2}\right)$, magnesium sulfate, silver nitrate, tetrachloroauric(III) acid (30\%), ethanol, hydroquinone (HQ), 2-propanol, ammonium hydroxide $30 \%$.

Applichem: polyvinylpyrrolidone K30 (PVP), glycerol, hydrochloric acid, trifluoroacetic acid (TFA), sodium citrate dehydrate, L-ascorbic acid, methanol, $N, N$-dimethylformamide (DMF).

ABCR: carboxyethylsilanetriol, sodium salt; 25\% in water.

\section{Methods}

UV-Vis extinction spectroscopy: Perkin Elmer, Lambda 35. Zetapotential: Malvern Zetasizer Nano Z. Transmission electron microscopy (TEM): Zeiss, EM 902. Scanning electron microscopy (SEM): Zeiss, Supra 50. SERS microscopy: WITec Alpha 
$300 \mathrm{R}$ confocal microscope with a Olympus $40 \times$ objective, a HeNe laser $\left(\lambda_{\mathrm{ex}}=632.8 \mathrm{~nm}\right)$, a monochromator with $30 \mathrm{~cm}$ focal length (600 grooves per mm grating) equipped with an EM-CCD (Andor, Newton).

\section{Acknowledgements}

Financial support from the European Union and the State of Lower Saxony (EFRE, project no. W2-80111700) is acknowledged. Thanks to Ulrich Krupp for access to FE-SEM and Bernd Walkenfort as well as Dennis Goesling for technical support.

\section{Notes and references}

1 V. Giannini, A. I. Fernández-Domínguez, S. C. Heck and S. A. Maier, Chem. Rev., 2011, 111, 3888.

2 R. Aroca, Surface-Enhanced Vibrational Spectroscopy, Wiley VCH, 2006.

3 E. Ringe, B. Sharma, A.-I. Henry, L. D. Marks and R. P. V. Duyne, Phys. Chem. Chem. Phys., 2013, 15, 4110.

4 C. L. Nehl, H. Liao and J. H. Hafner, Nano Lett., 2006, 6, 683.

5 E. C. L. Ru, P. G. Etchegoin and M. Meyer, J. Chem. Phys., 2006, 125, 204701.

6 R. Jin, Angew. Chem., Int. Ed., 2010, 49, 2826.

7 J. P. Camden, J. A. Dieringer, Y. Wang, D. J. Masiello, L. D. Marks, G. C. Schatz and R. P. Van Duyne, J. Am. Chem. Soc., 2008, 130, 12616.

8 J. M. McMahon, S. Li, L. K. Ausman and G. C. Schatz, J. Phys. Chem. C, 2012, 116, 1627.

9 E. L. Ru and P. Etchegoin, Principles of Surface-Enhanced Raman Spectroscopy and related plasmonic effects, Elsevier, 2008.

10 S. Schlücker, Angew. Chem., Int. Ed., 2014, 53, 4756.

11 S. Nie and S. R. Emory, Science, 1997, 275, 1102.

12 J. A. Dieringer, R. B. Lettan, K. A. Scheidt and R. P. Van Duyne, J. Am. Chem. Soc., 2007, 129, 16249.

13 M. Gellner, D. Steinigeweg, S. Ichilmann, M. Salehi, M. Schütz, K. Kömpe, M. Haase and S. Schlücker, Small, 2011, 7, 3445.
14 D. Craig, J. Simpson, K. Faulds and D. Graham, Chem. Commun., 2013, 49, 30.

15 L. Guerrini, F. McKenzie, A. W. Wark, K. Faulds and D. Graham, Chem. Sci., 2012, 3, 2262.

16 L. Guerrini and D. Graham, Chem. Soc. Rev., 2012, 41, 7085. 17 D.-K. Lim, K.-S. Jeon, H. M. Kim, J.-M. Nam and Y. D. Suh, Nat. Mater., 2010, 9, 60.

18 D. Graham, D. G. Thompson, W. E. Smith and K. Faulds, Nat. Nanotechnol., 2008, 3, 548.

19 A. S. D. S. Indrasekara, B. J. Paladini, D. J. Naczynski, V. Starovoytov, P. V. Moghe and L. Fabris, Adv. Healthcare Mater., 2013, 2, 1370.

20 B. Vlcková, M. Moskovits, I. Pavel, K. Sisková, M. Sládková and M. Slouf, Chem. Phys. Lett., 2008, 455, 131.

21 W. E. Doering and S. Nie, Anal. Chem., 2003, 75, 6171.

22 S. P. Mulvaney, M. D. Musick, C. D. Keating and M. J. Natan, Langmuir, 2003, 19, 4784.

23 Y. Wang and S. Schlücker, Analyst, 2013, 138, 2224.

24 C. Jehn, B. Küstner, P. Adam, A. Marx, P. Ströbel, C. Schmuck and S. Schlücker, Phys. Chem. Chem. Phys., 2009, 11, 7499.

25 Y. Wang, M. Salehi, M. Schütz, K. Rudi and S. Schlücker, Analyst, 2013, 138, 1764.

26 J. H. Yoon and S. Yoon, Langmuir, 2013, 29, 14777.

27 J. H. Yoon, Y. Zhou, M. G. Blaber, G. C. Schatz and S. Yoon, J. Phys. Chem. Lett., 2013, 4, 1371.

28 X.-L. Tang, P. Jiang, G.-L. Ge, M. Tsuji, S.-S. Xie and Y.-J. Guo, Langmuir, 2008, 24, 1763-1768.

29 W. Stöber, A. Fink and E. Bohn, J. Colloid Interface Sci., 1968, 26, 62 .

30 C. Graf, D. L. J. Vossen, A. Imhof and A. van Blaaderen, Langmuir, 2003, 19, 6693.

31 S. D. Perrault and W. C. W. Chan, J. Am. Chem. Soc., 2009, 131, 17042.

32 D. Steinigeweg and S. Schlücker, Chem. Commun., 2012, 48, 8682 .

33 D. Steinigeweg, M. Schütz and S. Schlücker, Nanoscale, 2013, 5, 110 . 